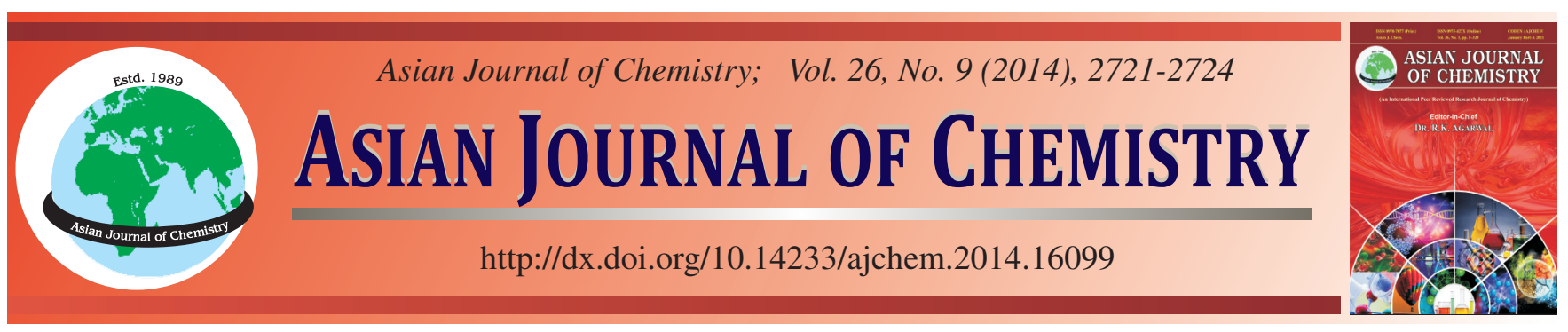

\title{
Synthesis and Mechanical Properties of Epoxy Composites Reinforced with Phosphazene Microspheres
}

\author{
ZhengPing Zhao*, JiAnBing Ji and Fengying Yu
}

Zhijiang College of Zhejiang University of Technology, Hangzhou 310024, P.R. China

*Corresponding author: E-mail: sjzhaolei@163.com

Using precipitation polymerization to synthesize polycyclotriphosphazene-sulfone microspheres with hexachlorocyclotriphosphazene and 4,4'-sulfonyldiphenol and filled epoxy matrix to prepare composites to study the thermal and mechanical modified mechanisms. Polycyclotriphosphazene-sulfone microspheres has an average particle diameter of $500 \mathrm{~nm}$, the initial decomposition temperature is $457.5^{\circ} \mathrm{C}$ and the solid residual rate at $600{ }^{\circ} \mathrm{C}$ is $63.1 \%$. The tensile strength $(55.95 \mathrm{MPa})$, impact strength $\left(25.46 \mathrm{KJ} / \mathrm{m}^{2}\right)$, flexural strength (140.65 MPa), elongation at break (38.6\%) of the EPM-3 composite material are 1.44, 2.74, 1.61, 1.89 times of EPM-0, respectively. Polycyclotriphosphazene-sulfone microspheres uniformly dispersed in the epoxy matrix and effectively improve the $\mathrm{T}_{\mathrm{g}}$ and thermostability of the composites.

Keywords: Polycyclotriphosphazene-sulfone microspheres, Synthesis, Composites, Mechanical property.

ᄂ - - - - - - - - - - - - - - - - - - - - - - - - -

\section{INTRODUCTION}

Polyphosphazene as an organic-inorganic hybrid polymer is a class of novel macromolecules containing alternate phosphorus-nitrogen single and double bonds with two changing organic side groups to be prepared new specific functions materials. Because the phosphorus-nitrogen bonds are extremely flexible due to the low torsional energy and a large variety of side groups, polyphosphazenes can be made with a wide range of chemical and physical properties, like excellent heat resistance, cold-resistant, water-tolerant, solvent resistance, radicalization resistance and flame retardant performances in many $\operatorname{areas}^{1-12}$.

Polyphosphazene microspheres attracted more and more attention at biomedicine and catalyst carrier areas, because of its structure designed, particles size and shape controllable and high surface tension. Surface hydroxyl-rich microspheres lead to our attentions due to 3 main reasons. (1) Preparation of the microspheres with the surface rich in reactive hydroxyl groups is simple, economical and low-cost. (2) This preparation method does not require any stabilizer. (3) Hydroxyl microspheres have excellent thermal stability. In addition, nanomicrospheres will play a key role in many areas. Therefore, we design to prepare polymer-polyphosphazene microspheres to improve thermal stability and mechanical properties.

In recent years, there is a great need for composites because the combination of two or more materials can lead to enhance performance and outstanding properties compared to their constituents $^{13}$. Many efforts have been made to develop the inorganic particles as additives for modification of epoxy composites, while no relevant reports of these in the field of polyphosphazene microspheres. In this work, polycyclotriphosphazene-sulfone microspheres were synthesized and compounded it with epoxy (EP-51) subsequently. Ultimately, thermal and mechanical behaviors of composites with different polycyclotriphosphazene-sulfone microspheres contents were investigated, which would be important and meaningful for polyphosphazene derivatives in the field of synthesis and application in different fields.

\section{EXPERIMENTAL}

Hexachlorocyclotriphosphazene (HCCP) (synthesized as described in the literature ${ }^{14}$ ) was recrystallized from dry hexane followed by sublimation $\left(60{ }^{\circ} \mathrm{C}, 0.05 \mathrm{mmHg}\right)$ twice before use (m.p. $\left.=112.5-113^{\circ} \mathrm{C}\right)$. Acetone, acetonitrile, 4,4'-sulfonyldiphenol and triethylamine were obtained from Sinopharm Chemical reagent Co., Ltd (Shanghai China). Epoxy resin (EP51) was purchased from Shanghai resin Co., Ltd (Shanghai China) and its average epoxy value is 0.51 (the term 'epoxy value' represents the fractional number of epoxy groups contained in $100 \mathrm{~g}$ of resin). All glassware was dried in an oven under vacuum before use.

FT-IR spectra of all samples were recorded using polymer granule on a Perkin-Elmer Wellesley MA spectrophotometer. 
Thermogravimetric analysis was performed on a thermogravimetric analysis 7 instrument (Perkin-Elmer) thermal analysis system. Sample weight taken was 2-4 mg. DSC analysis was carried out on a Perkin-Elmer Pyris 2 DSC analyzer (PerkinElmer) and the glass transition temperature of the polyamides is measured during the heating part of the second thermal cycle at a heating rate of $10^{\circ} \mathrm{C} / \mathrm{min}$ in $\mathrm{N}_{2}$ atmosphere. Sample weight taken was $15-20 \mathrm{mg}$. Wide-angle X-ray scattering measurements (XRD) were performed on a Bruker AXS-D8 Avance $\mathrm{X}$-ray diffractometer with a copper target $(40 \mathrm{kV}, 15 \mathrm{~mA})$. The microstructures of samples were recorded using a Cambridge S250MK3 scanning electron microscope (SEM, U.K.). The tests of mechanical properties were performed on Shimadzu DZ10KN tensile machine.

Synthesis of polycyclotriphosphazene-sulfone microspheres: Triethylamine $(4 \mathrm{~mL}, 0.0287 \mathrm{~mol})$ was added to a solution of 4,4'-sulfonyldiphenol (1.6 g, $0.006 \mathrm{~mol})$ and hexachlorocyclotriphosphazene $(0.8 \mathrm{~g}, 0.002 \mathrm{~mol})$ in acetonitrile $(200 \mathrm{~mL})$. The reaction mixtures were stirred in an ultrasonic bath $(50 \mathrm{~W}, 40 \mathrm{~Hz})$ at $40{ }^{\circ} \mathrm{C}$ for $1 \mathrm{~h}$. The resultant particles were obtained by centrifugation and then were washed three times using deionized water and acetone, respectively. The solid was dried under vacuum at $50{ }^{\circ} \mathrm{C}$ for $24 \mathrm{~h}$ to yield polycyclotriphosphazene-sulfone microspheres directly as a hard white powder.

Preparation of epoxy/polycyclotriphosphazene-sulfone microspheres composites: The epoxy resin (epoxy-51) was mechanically mixed with polycyclotriphosphazene-sulfone microspheres at different content $(0,1,2,3,4$ wt. \%) in a beaker at $80^{\circ} \mathrm{C}$ for $40 \mathrm{~min}$ to evaporate acetone and then the temperature increased to $120^{\circ} \mathrm{C}$ for $20 \mathrm{~min}$. When the mixture was naturally cooled to $30^{\circ} \mathrm{C}$, the hardener diethylenetriamine was added with mechanically mixing for $5 \mathrm{~min}$. Finally, the mixture was poured into virgate molds $(\Phi=5.7 \mathrm{~mm}, 1 \approx 123 \mathrm{~mm})$, followed by curing at $80^{\circ} \mathrm{C}$ for $1 \mathrm{~h}$ and $100^{\circ} \mathrm{C}$ for $1 \mathrm{~h}$ and then the epoxy/polycyclotriphosphazene-sulfone microspheres composites (EPM-0, EPM-1, EPM-2, EPM-3, EPM-4, respective) were obtained.

\section{RESULTS AND DISCUSSION}

Structure of polycyclotriphosphazene-sulfone microspheres: The chemical structure of the polycyclotriphosphazenesulfone microspheres was confirmed by FT-IR as shown in Fig. 1. The band at $1478 \mathrm{~cm}^{-1}$ and $1586 \mathrm{~cm}^{-1}$ corresponds to the benzene ring, $1260 \mathrm{~cm}^{-1}$ and $1200 \mathrm{~cm}^{-1}$ are assigned to $\mathrm{P}=\mathrm{N}$ groups, $940 \mathrm{~cm}^{-1}$ is due to $\mathrm{P}-\mathrm{O}-\mathrm{Ar}$ groups and the characteristic peaks for $\mathrm{O}=\mathrm{S}=\mathrm{O}$ can be seen at $1290 \mathrm{~cm}^{-1}$. The results demonstrate that the polycyclotriphosphazene-sulfone microspheres have the expected chemical structures.

The morphology of the polycyclotriphosphazene-sulfone microspheres were characterized by SEM are shown in Fig. 2. The lower-magnification image showed that the polycyclotriphosphazene-sulfone microspheres were uniform grain spherical material. The higher- magnification image showed that the polycyclotriphosphazene-sulfone microspheres was about $500 \mathrm{~nm}$ in diameter and has rough surface and extremely clean.

Thermal properties of polycyclotriphosphazenesulfone microspheres and epoxy/polycyclotriphosphazenesulfone microspheres composites: Thermal properties of

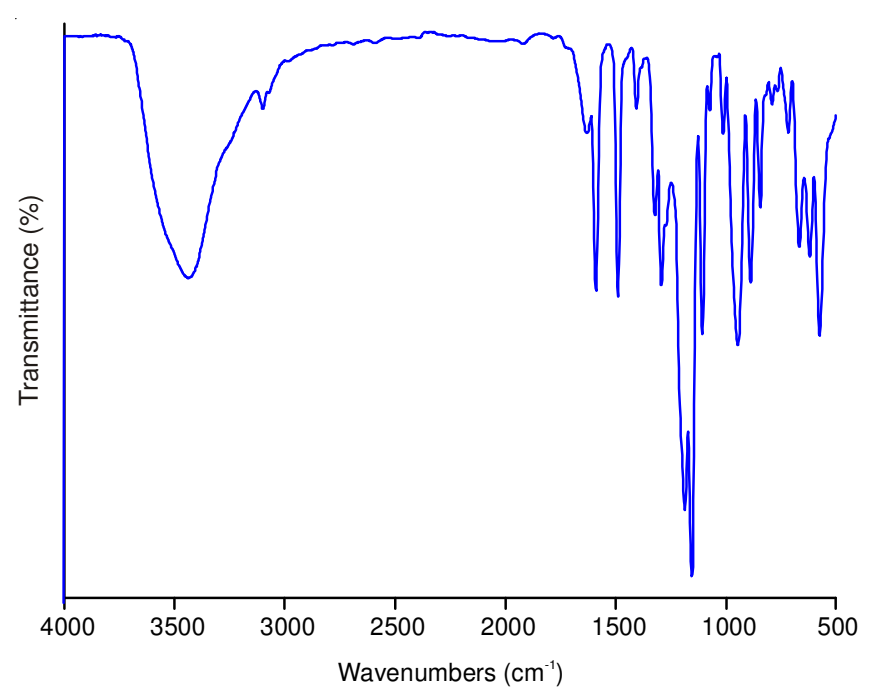

Fig. 1. FT-IR spectrume of the polycyclotriphosphazene-sulfone microspheres
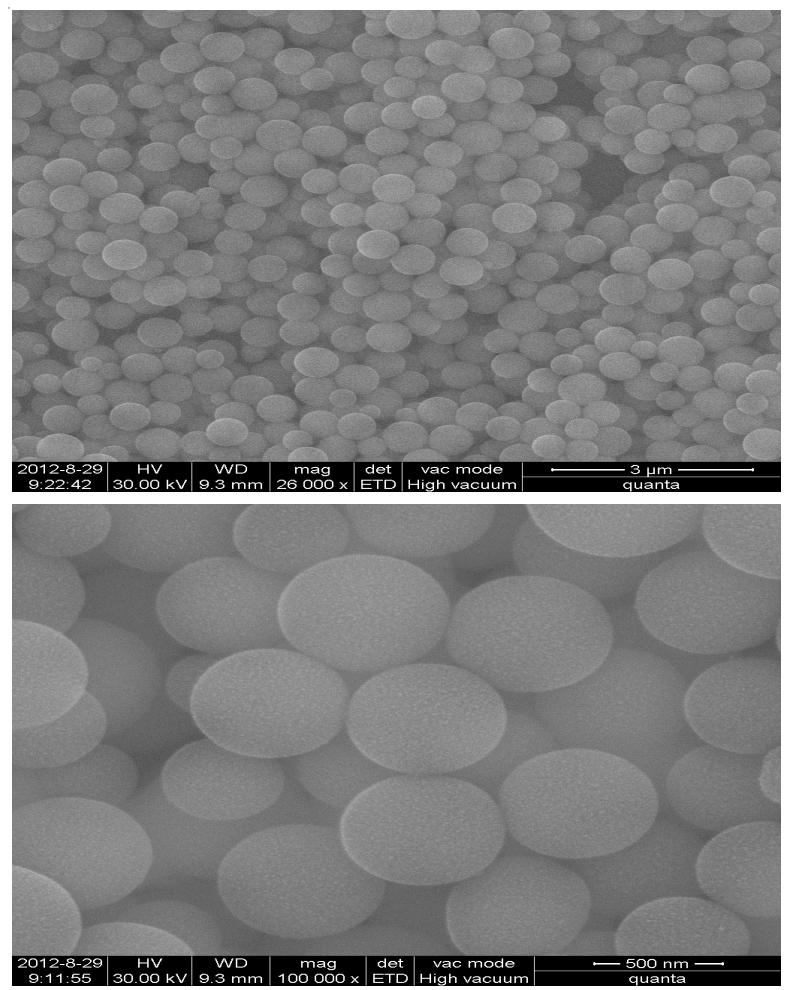

Fig. 2. SEM photographs of the polycyclotriphosphazene-sulfone microspheres

polycyclotriphosphazene-sulfone microspheres and epoxy/ polycyclotriphosphazene-sulfone microspheres composites were examined by inverstigating the glass transition temperature $\left(\mathrm{T}_{\mathrm{g}}\right)$ and thermal stability are shown in Figs. 3 and 4 , respectively. The glass transition region shifted towerd a higher termperature with the increasing polycyclotriphosphazenesulfone microspheres content. The temperature of EPM-0 was about $82.1^{\circ} \mathrm{C}$, in comparison with it, the temperature of EPM3 increased by $9.5^{\circ} \mathrm{C}$. A little polycyclotriphosphazene-sulfone microspheres microspheres uniformity disperse in the composites and the epoxy resin molecular chains wrapped around the microspheres to form a solid reinforced frame, which can effectively limit the movement of the polymer chain at heating 
condition, thereby improving the temperature. Meanwhile, the large number of hydroxyl-rich surface of the microspheres can form hydrogen bonds with molecular chains to enhance the interface combination between the microspheres and epoxy resin. Polycyclotriphosphazene-sulfone micros-pheres play a role in chemical crosslinking points, increase the crosslike density and improve the temperature. When an excess amount of microsphere filled, the particles agglomeration increased and leaded to the interface incorporation reduced between the particles and matrix which due to the reducing of the temperature.

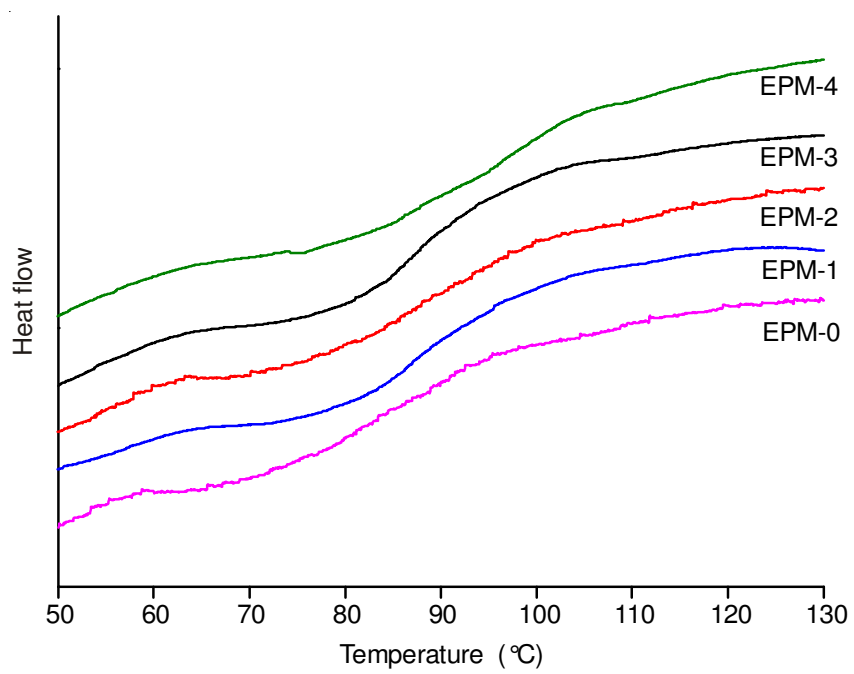

Fig. 3. DSC curves of epoxy/polycyclotriphosphazene-sulfone microspheres composites

According to Fig. 4, $\mathrm{T}_{0}$ of the EPM-4 occurred at about $328.9^{\circ} \mathrm{C}$, which was $10^{\circ} \mathrm{C}$ higher than EPM-0 $\left(\mathrm{T}_{0}=318.3^{\circ} \mathrm{C}\right)$ and char residue rate of the EPM- 4 at $600{ }^{\circ} \mathrm{C}$ was 14.4 , which was much higher than EPM-0 (char rate $=10.5 \%$ ). It is indicated that the thermal stability of the composites was higher then pure epoxy. The significantly improved thermal stability of the composites could be attributed to adding polycyclotriphosphazene-sulfone microspheres. polycyclotriphosphazenesulfone microspheres consisted of inorgnic cyclotriphosohazene rings in the fully cross-linking structures which inpart excellent

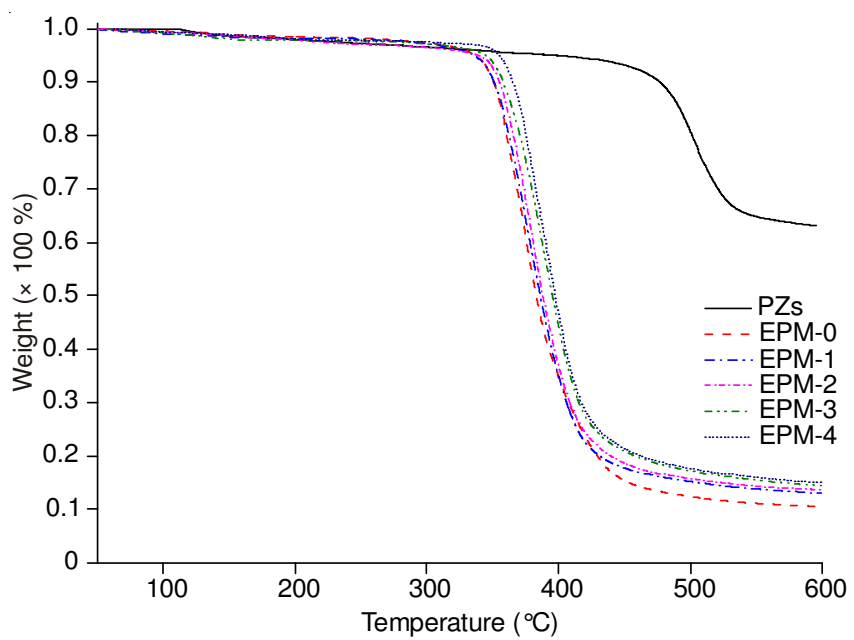

Fig. 4 Thermogravimetric analysis curves of epoxy/polycyclotriphosphazene-sulfone microspheres composites thermal stability (Fig. 4). In addition, the cross-linking structure of the composites could quickly transfer the heat from the epoxy matrix to the polycyclotriphosphazene-sulfone microspheres to ensure that the heat distribution is uniform through the composites, avioding that the materials are ahead decomposed due to excessive heat in local.

Mechanical properties of epoxy/polycyclotriphosphazenesulfone microspheres composites: The results of tensile measurements were listed in Table-1. From the table, a significant increase in the tensile strength, impact strength, flexural strength and elongation at break when compared to that of unfilled EPM-0 is observed. However, we can see that they increase up to a polycyclotriphosphazene-sulfone microspheres content of $3 \mathrm{wt} . \%$, followed by a decrease with increasing the content. Generally, using common fillers to reinforce composites, the strength can be effectively improved, but the elongation at break usually has a remarkable decrease. For the composites, improvement in strength and elongation were obtained. It may be attributed to cross-linking structure of the composites. The cross-linking structure could quickly transfer the tensile strength from the epoxy matrix to the full crosslinking polycyclotriphosphazene-sulfone microspheres, then the polycyclotriphosphazene-sulfone microspheres could be deformed to distribute strength through the composites, avoiding that the materials are ahead fracture due to excessive strength in local.

\begin{tabular}{ccccc}
\multicolumn{5}{c}{ TABLE-1 } \\
$\begin{array}{c}\text { MECHANICAL PROPERTIES OF EPOXY } \\
\text { AND EP/PZs COMPOSITES }\end{array}$ \\
\hline CODE & $\begin{array}{c}\text { Tensile } \\
\text { Strength } \\
(\mathrm{MPa})\end{array}$ & $\begin{array}{c}\text { Impact } \\
\text { Strength } \\
\left(\mathrm{kI} / \mathrm{m}^{2}\right)\end{array}$ & $\begin{array}{c}\text { Flexural } \\
\text { strength } \\
(\mathrm{MPa})\end{array}$ & $\begin{array}{c}\text { Elongation } \\
\text { at break } \\
(\%)\end{array}$ \\
\hline EPM-0 & 38.76 & 9.28 & 87.13 & 20.42 \\
EPM-1 & 43.42 & 19.63 & 97.05 & 32.15 \\
EPM-2 & 49.42 & 23.29 & 123.79 & 35.73 \\
EPM-3 & 55.95 & 25.46 & 140.65 & 38.60 \\
EPM-4 & 52.44 & 26.11 & 111.45 & 38.95 \\
\hline
\end{tabular}

The dispersion of polycyclotriphosphazene-sulfone microspheres greatly affects the properties of the composites. In order to study the toughening mechanism of nanoparticles in composites, the morphologies of the fracture surface of EPM-3 specimen which was obtained by brittle fracture of bar sample in liquid nitrogen were examined by SEM as shown in Fig. 5. The polycyclotriphosphazene-sulfone microspheres are dispersed well in the epoxy matrix, showing that the polycyclotriphosphazene-sulfone microspheres have a good compatibility with the epoxy matrix. It is noteworthy that polycyclotriphosphazene-sulfone microspheres disperse more uniformly in the epoxy resin, have a good interfacial adhesion and firmly embedded in the base materials. There is excessive layer between microspheres and matrix resin. The main reasons are, on the one hand, polyphosphazene microspheres having strong surface tension and good surface bond strength with polar epoxy matrix, other hand, the oxygen atom in the epoxy prone to take place hydrogen bond crosslinking with the polyphosphazene microspheres which enriched in oxygen atoms and further enhance the bonding strength.

In order to study the toughening mechanism of microspheres in epoxy composites, the morphology of the fracture 

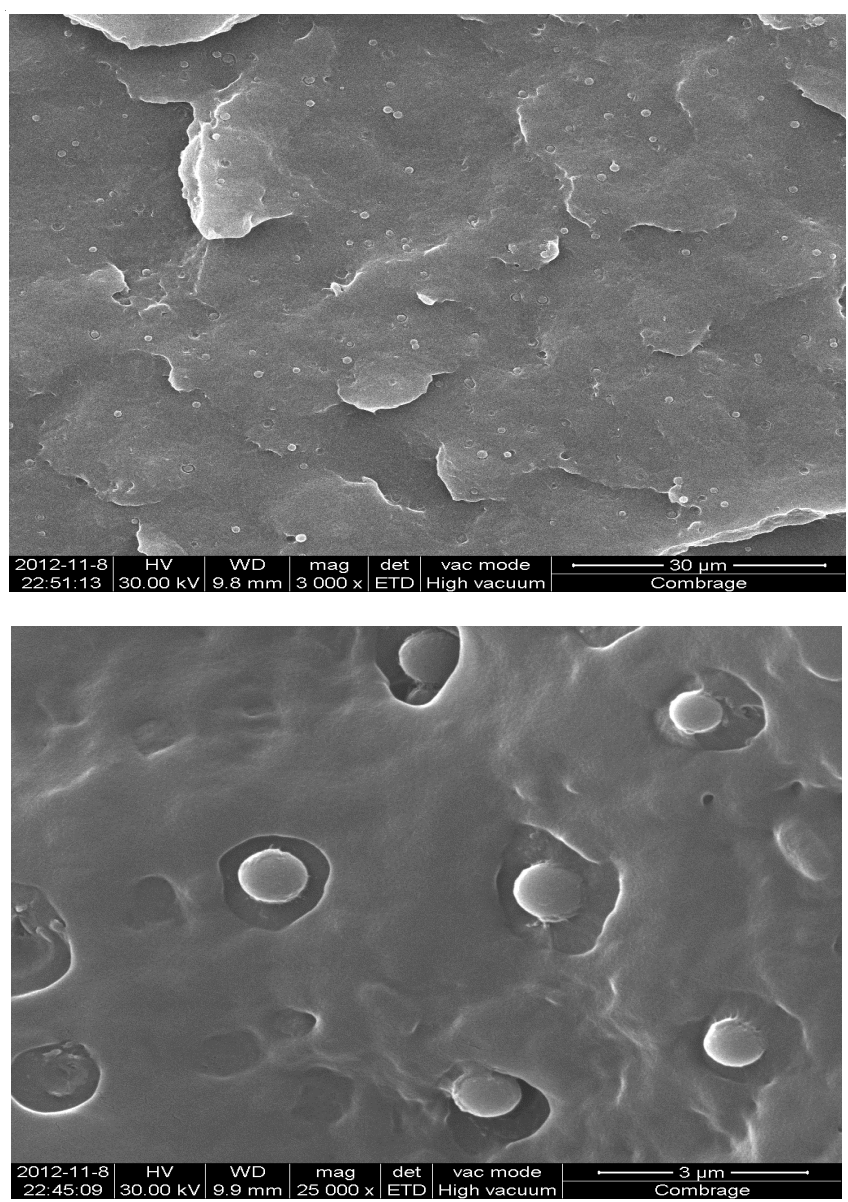

Fig. 5. Brittle fracture morphology of EPM-3 composite

surface of specimens were examined by SEM as shown in Fig. 6. (a) is the pure epoxy tensile fracture surface. The cross section is smooth, single crack direction and linear expansion, showing a clear brittle fracture characteristic. (b) is the tensile fracture surface of epoxy composite modified by $3 \mathrm{wt} . \%$ polycyclotriphosphazene-sulfone microspheres. There are different sizes of dimples on the fracture surface and the tension close to the critical crack extension force acting on the crack tip region, the matrix deformation showing ductile fracture morphology is typical of ductile fracture. Combined with the tensile data, it is shown that the microspheres can effectively improve the fracture toughness of epoxy resin, which has similar toughening mechanism as inorganic nanoparticles and elasticity particles.

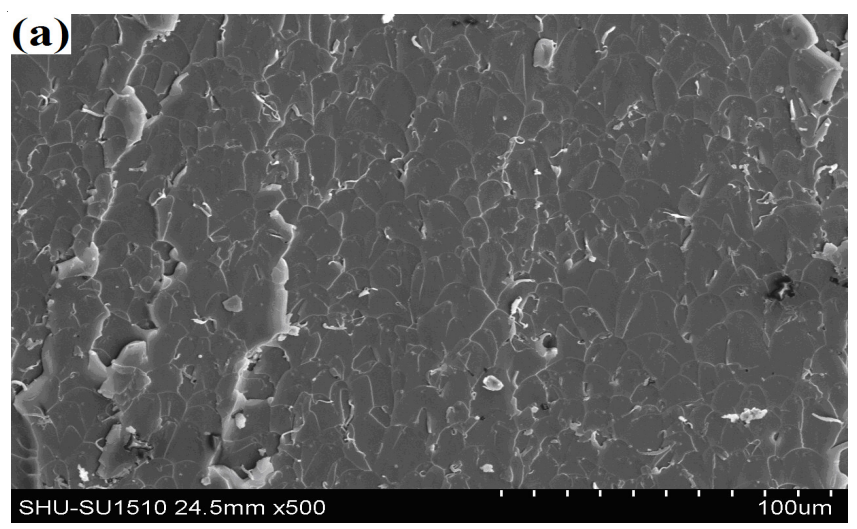

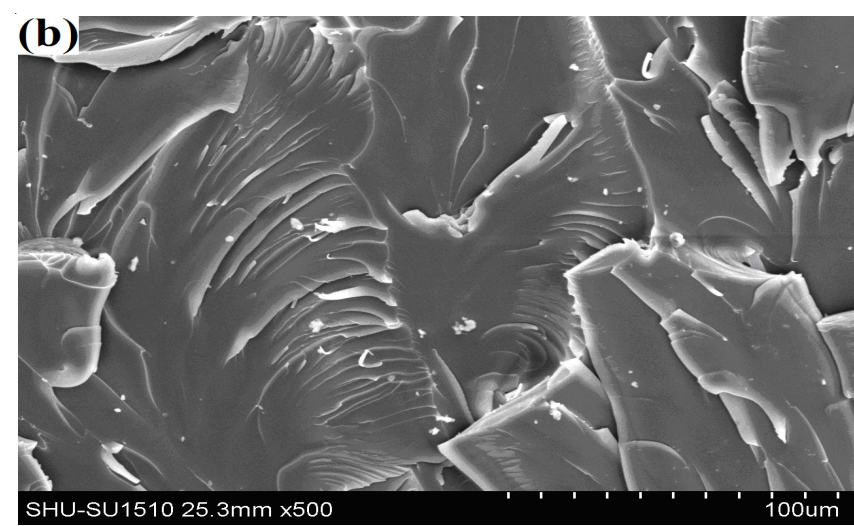

Fig. 6. Tensile fracture microstructure of epoxy and composites

\section{Conclusion}

Using precipitation polymerization one step to synthesize polyphosphazene nanospheres (polycyclotriphosphazene-sulfone microspheres) with HCCP and 4,4'-sulfonyldiphenol and filled epoxy composite materials to study the mechanical mechanism. Polycyclotriphosphazene-sulfone microspheres has an average particle diameter of $500 \mathrm{~nm}$ and the excellent thermal stability. The initial decomposition temperature of the polycyclotriphosphazene-sulfone microspheres is $457.5^{\circ} \mathrm{C}$ and the solid residual rate at $600{ }^{\circ} \mathrm{C}$ is $63.09 \%$. polycyclotriphos-phazenesulfone microspheres disperse more uniformly in the epoxy resin, have a good interfacial adhesion and firmly embedded in the base materials. Polycyclotriphosphazene-sulfone microspheres enhance temperature and thermal stability of the composites. For all Epoxy composites, 3 wt. \% content of polycyclotriphosphazene-sulfone microspheres filled, the mechanical properties were strengthened. The tensile strength of the composite material (EPM-3, 55.95 MPa) is 1.44 times that of EPM0 , impact strength (EPM-3, 25.46 KJ/m²) is 2.74 times that of EPM-0, flexural strength (EPM-3, $140.65 \mathrm{MPa}$ ) is 1.61 times that of EPM-0, elongation at break (EPM-3, 38.6\%) is 1.89 times for EPM-0.

\section{REFERENCES}

1. K.M. Abraham, M. Alamgir and S.J. Perrotti, J. Electrochem. Soc., 135, 535 (1988).

2. H.R. Allcock, in E.J. Glass, Water Soluble Polyphosphazenes and thier Hydorgels, In: Hydrophilic Polymers, Adv. Chem. Ser., 248, 3 (1996).

3. H.R. Allcock, C.G. Cameron, T.W. Skloss, S. Taylor-Meyers and J.F. Haw, Macromolecules, 29, 233 (1996).

4. H.R. Allcock, C.A. Crane, C.T. Morrissey, J.M. Nelson, S.D. Reeves, C.H. Honeyman and I. Manners, Macromolecules, 29, 7740 (1996).

5. H.R. Allcock and W.E. Krause, Macromolecules, 30, 5683 (1997).

6. H.R. Allcock, W.R. Laredo and R.V. Morford, Solid State Ion., 139, 27 (2001).

7. H.R. Allcock, J.M. Nelson, R. Prange, C.A. Crane and C.R. de Denus, Macromolecules, 32, 5736 (1999).

8. H.R. Allcock, E.S. Powell, A.E. Maher, R.L. Prange and C.R. de Denus, Macromolecules, 37, 3635 (2004).

9. S.J. Coles, D.B. Davies, R.J. Eaton, A. Kiliç, R.A. Shaw and G.Y. Çiftçi, Polyhedron, 25, 953 (2006).

10. P. Selig, E. Herdtweck and T. Bach, Eur. Chem. J., 15, 3509 (2009).

11. R.J. Waltman, N. Kobayashi, K. Shirai, A. Khurshudov and H. Deng, Tribol. Lett., 16, 151 (2004).

12. Y. Zhu, X.B. Huang, J.W. Fu, G. Wang and X. Tang, Mater. Sci. Eng., 153, 62 (2008).

13. E. Sevkat, B. Liaw, F. Delale and B.B. Raju, Compos., 40, 1090 (2009).

14. K. Horie, Y. Morita, Y. Mikamori, M. Suzuki and S. Yano, US Patent 4382914 (1983). 